\title{
Automated data collection for observed events
}

\author{
LAWRENCE J. FITZPATRICK \\ University of Minnesota, Minneapolis, Minnesota 55455
}

\begin{abstract}
This paper presents the general requirements for dealing with the temporal aspects of multiple binary events. Discussions of the need for common data structures in this work and the optimal structures for efficient storage of this type of data are also presented. General features of automated data collection devices and a description of the data input device used by the author are detailed. Finally, a comparison is made between a number of data input devices.
\end{abstract}

A number of different classification schemes and keyboard recording devices are currently available for categorizing and collecting observational data (Hopf, 1972; Odom, Mitchell, \& Lindberg, 1970; Sackett, Stephenson, \& Ruppenthal, 1973; Stephenson, Smith, \& Roberts, 1975; White, 1971; Hollenbeck, Smythe, \& Sackett, Note 1; Holm, Note 2). Given a user-defined set of events, most researchers would agree that all of the information required for their data analyses can be collected by recording (1) the time of onset and offset of each event and (2) the event that is referred to by each time. Input devices and classification schemes typically utilize all or part of the nominal and temporal information (Bakeman, Note 3).

Agreement is not difficult with respect to the recording task, but the issue of what specifically to commit to the permanent data record is far more volatile, reaching into the areas of classification schemes, modes of entry, and methods of representing time and events in the storage medium. Classification schemes are quite numerous and often idiosyncratic to specific researchers. The act of classification and subsequent establishment of hierarchical rules for recording events is a conceptual endeavor rightfully reserved for the individual researcher or determined by the conceptual framework which he represents. The investigator should not be constrained in this act by his recording hardware.

\section{NEED FOR A COMMON DATA STRUCTURE}

The above notwithstanding, it is of great interest to develop agreement on a common structure for the data. Agreement at this point will permit direct communication between researchers, such that diverse individuals

Support for preparation of this manuscript and for early research into data input devices was provided by Program Project Grant 1 PO HD 05027 from the National Institute of Child Health and Human Development, granted in part to Willard W. Hartup and William R. Charlesworth, Institute of Child Development, University of Minnesota. I wish to express my sincere appreciation to Gordon Stephenson, University of Wisconsin, for commenting on an early draft of this paper. Requests for reprints should be sent to Lawrence J. Fitzpatrick, Institute of Child Development, 51 East River Road, University of Minnesota, Minneapolis, Minnesota 55455. can study common data or select from a broad range of editing and analysis packages developed in individual laboratories. More important, it will enhance outside scientific validation and refutation of work from one laboratory by minimizing the need to rewrite analysis programs or translate data at a second laboratory in order to restudy the problem.

Agreement on a common structure for recording data at this level becomes much easier if one conceptually separates the event from the scheme by which it is classified. Whether the event is a simple recognizable act or a conjunction of several categorical conditions, it is possible to represent it as a unit with specific onset and offset times; the name of that unit can be most simply represented as a number from 1 to $\mathrm{N}$. When the subsequent data analysis task is broadened to include event selection, logical event combination, and event naming (Fitzpatrick, 1977), it becomes possible to establish or recreate hierarchical event structures from a simple time-event format.

\section{BASIC MODES OF KEYBOARD ENTRY}

A potential source of difficulty in implementing a common data structure stems from differences in the two basic modes of entering observations on keyboard recording devices. The two basic modes, parallel and serial input, optimize different aspects of data collection and result in a qualitative difference with which the observations are classified or temporally located in the record.

Parallel data input logically allows more than one event to be recorded at a time while requiring the observer to monitor a momentary-contact-type key at each fingertip for each event to be recorded, depressing the key when the event begins and releasing the key when it ends. Parallel input requires that the observer remember which event a given finger represents, that he press down with that finger while the event is occurring, and that his fingers operate independently. Serial input generally utilizes a numerical keyboard on which only one character can be recognized at a time. Typically, when an event begins, the observer enters a numerical code and, when it ends, he reenters the code plus an 
additional signal to end the event. Serial input requires that the observer remember the code for each observed event and then enter that series of characters on the input keyboard. Usually each code is stored along with its time of entry.

Parallel and serial input modes appear to serve complementary functions. Event timing can be very accurately kept in the parallel mode since the observer has 10 or fewer events (a relatively small number of events) to monitor at a time and he can instantly depress additional keys when additional events occur. When a large number of events are to be studied, the parallel mode works well with multiple passes through videotaped behavioral records. Multiple passes allow accumulation of accurate temporal records on many events but cost additional observation time. A synchronizer signal is used to permit temporal integration of the different passes through the same videotaped record. The parallel input mode is effective with naive observers, requiring only 3 or $4 \mathrm{~h}$ of practice to obtain reasonable reliability with readily observable events. On the other hand, the serial input mode appears optimal in a field setting where trained observers must record a large number of events in a single pass. The gain in number of units per single observation session is offset by a loss in timing accuracy since multiple events occurring simultaneously must be entered one at a time and the observer must also remember to enter the offset of each event. The latter, of course, is an observer problem rather than a hardware limitation.

\section{CONSIDERATIONS FOR EFFICIENT DATA STORAGE}

A common data structure that retains onset and offset times for each occurrence of an event should be chosen on the basis of efficiency of data storage, since storage represents a major expense in most computer systems. In most cases, storage efficiency correlates positively with computer time required to transform individual data to and from the common structure, so efficient data storage should also result in efficient data translation. The time and event components of the data format will be discussed separately.

The time of an event in the record can be represented as cumulative time from the beginning of the record or as time elapsed since the last entry. Use of elapsed time permits a smaller time record with no loss of information. For efficient storage, tradeoffs involving size of the time word and event representation are somewhat more complex. Convenient "byte" sizes for time and event character representation are 6 and 8 bits, with 6-bit (64-character capacity) stored efficiently on seventrack magnetic tape and 8-bit (256-character capacity) efficiently stored on nine-track tape. Algorithms to deal with both byte sizes are available on most large computer systems. For efficient storage, time representa- tion should be some multiple of the optimal byte size. A small-capacity time word requires many repetitions of the same line to represent record lines with long duration; a large-capacity time word wastes space at each line. It is recommended that time be stored as a fixed-length integer in tenths of seconds and that the word size for 6-bit representation be 2 bytes long (12-bit or 409.5-sec capacity). Word size for 8-bit representations should be 1 byte long (8-bit or $25.5-\mathrm{sec}$ capacity).

Three basic storage structures that retain the necessary temporal information are: (1) line-by-line entry of the elapsed time that each particular subset of events occurred and a single bit to represent the occurrence or nonoccurrence of each event studied; (2) the same lineby-line structure, but instead of sequenced bit representation for each event, a list of the numbers representing the events occurring in each line and a stop character indicating the end of a record line; (3) the same line-byline structure but only a list of the numbers representing events that changed state at the end of the line.

Choice of optimal event representation can be made in two steps. First, the most efficient character representation is determined and then it is compared with sequential bit representation. When the events currently occurring are represented (Type 2), the number of events expected on a given line increases exponentially with increases in the total number of possible events. A similar increase in possible events, when event changes are represented (Type 3), produces only a small increase in the probability that more than one event will occur on any record line. Furthermore, if a time word is followed by only one event word in entries represent. ing event changes and separate entries are made when more than one event changes at a time, a code which indicates the end of a record line is not necessary. Therefore, event change representation is preferred for large records. The break-even point in record length between the two methods of representation should fall well below 20 events, so event change representation should be the better character representation for all except the smallest records.

Sequential bit representation (Type 1) uses only the number of bits for which there are events to be represented; event change character representation (Type 3) uses 6 or 8 bits/character plus, at the most, 2 additional bits to allow for those occasions when more than one event changes at a time. Thus, records with more than 8 possible events in 6-bit mode or 10 possible events in 8-bit mode will be more efficiently stored using event change character representation.

To summarize, the candidate data structure that should prove most efficient of space in all but the smallest records would utilize a series of fixed-length time integers followed by one event character each. The exact size of the integer-character units is dependent upon whether 6- or 8-bit bytes are the optimal byte size 
for character manipulation and storage. For 6-bit character bytes the integer-character unit should contain a 12-bit time integer followed by a 6-bit character; for 8-bit character bytes the integer-character unit should contain an 8-bit time integer and an 8-bit character.

\section{CRITERIA FOR ASSESSING DATA RECORDING DEVICES}

Any device that stores events along with their times of onset and offset produces adequate information to allow restructuring into a common data format. Of the two basic types of data input devices, one can handle a large number of separate units throughout an observation session but can enter events serially only one at a time; the second can handle a limited number of events in one session but can record more than one event at a time in parallel. (Of course, a single device can be used both ways; see Table 1.) Important criteria for assessing input devices include the completeness of the time-event information which they can provide, the type of memory used to store data, the amount of information they can record before reaching capacity, availability of a paper copy of the input record for correction, verification and backup, the portability and remote recording capability of the device, provisions to allow synchronization of separate data records, and the method used to transmit data and speed of transmission to a computer. The first two criteria have been discussed earlier; the rest deserve attention at this point.

Memory considerations involve permanence of the data record on the storage medium, data storage capacity, and availability of a paper copy of the data record. Data can be stored in electronic memory, on magnetic tape or disk, or on paper tape or punched cards. Input devices that use one or more of these types of storage are available. A paper copy provides very good backup for all electronic systems, providing a record that can be used to correct any storage or transmission error, including data loss which may occur anywhere in the data entry process. Probability of storage and transmission errors is lowest with electronic memory and highest with simple audio cassette interfaces.

Disk and magnetic tape contain practically unlimited storage, while electronic memory is normally purchased in modules. Small disk systems and computer-grade cassette tape systems are somewhat expensive but can hold one-half million or more bits of information. Simple audio cassette systems can contain the equivalent of one-quarter million bits of information or more on a 30-min cassette tape if the data is stored in an efficient format. Using an optimal data format, a new event introduced every $5 \mathrm{sec}$ utilizes 23,040 bits, or 2,880 8 -bit bytes of memory per hour. Storage of complex entry codes, use of larger time bytes, or less efficient entry formats increase the amount of storage required. When capacity limit is reached, the user must either exchange the memory or transfer his data to a more permanent facility; only after this can he reuse the memory. Disk and tape memories can readily be exchanged but electronic memory normally requires readout and reuse.

Permanence of the stored record is also important. The contents of standard electronic memory are lost whenever power is removed, but many input devices using this type of storage provide backup power to retain the information in memory when the input device is no longer actively used. Disk, tape, and punched paper memory have the advantage of permanence and require no power to retain the information, but punched paper is often inconvenient to store and is not reusable.

Portability involves three separate factors: (1) accessibility to timesharing and independence of the device from a specific dedicated host computer for reading data generated by the device, (2) weight and ease of handling of the device, and (3) the ability to operate in locations away from conventional power sources and the length of time continuous operation is possible in the isolated state. Battery operation and light weight are not difficult to obtain. Most newer devices designed specifically for behavioral data logging are both lightweight and battery operated.

Provision to allow synchronization and, consequently, merging of several data records is important to users who employ multiple observers and input devices concurrently to record data or to those whose methods call for multiple passes through videotaped records to capture the required data. This function has not been widely automated but will be as users discover its utility.

Finally, method and speed of data transmission to the storage and analysis facility is of considerable interest. Use of timesharing ports to transmit data is probably most flexible, since these ports normally utilize telephone communication and data entry from many locations is possible. Transmission speed may also be important, especially in long projects. Systems using electronic memory, disk, or computer-grade cassette storage should have fast transmission rates, while audio cassette storage somewhat restricts maximum transmission speed. Punched paper storage is normally slow. Systems employing punched cards are limited to batch input facilities; punched paper tape storage can utilize Teletypes or high-speed tape readers for data entry.

\section{ELOG DATA ACQUISITION SYSTEM}

Before classifying some currently available data recording devices, the parallel input data entry device ELOG, developed with the BEHAVE data analysis system (Fitzpatrick, 1977), will be described. Figure 1 presents the hardware configuration for ELOG data recording. This system utilizes a series of hand-held or tabletop five-key "keyboards" which plug directly into a battery-operated data input microprocessor along with 
A. DATA RECORDING -- DATA INPUT MODE

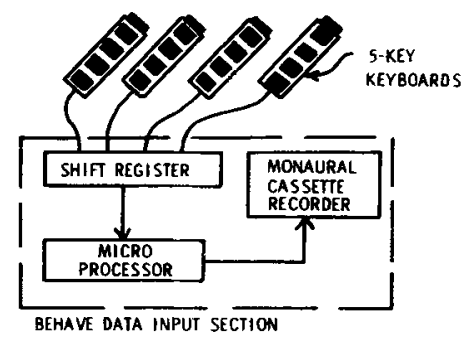

B. DATA RECORDING - DATA TRANSMISSION MODE

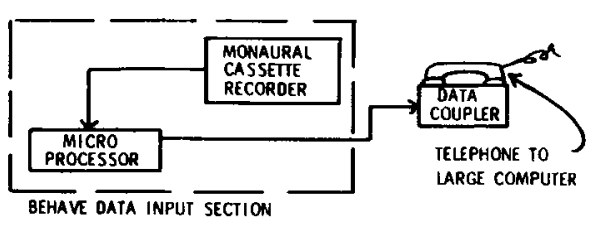

Figure 1. ELOG data acquisition hardware.

an audio tape cassette data storage device. The user begins data collection by assigning a specific event to each utilized key of each input keyboard. He then positions the cassette at its starting location, sets the input device to "record," and turns on the microprocessor. When he is ready to record, he turns on the "time" switch and begins. When an event occurs in the behavioral domain, the observer depresses the appropriate key; when the event terminates, he releases the key. An occasional 3,000-Hz "beep" signal can be fed to the input device through a separate data channel to provide a synchronization signal for later merging of data files. The observer can simultaneously record all keyboard keys. The "time" switch is turned off to terminate a data run. More than one observer can record data simultaneously by connecting their keyboards into the 20-event microprocessor. A maximum of four input keyboards can be connected to the remote input unit. To transmit data to a computer for analysis, the user normally connects his ELOG processor to a data coupler, sets the cassette containing data to be read at its starting location, sets the data unit to "transmit," logs in on the timesharing facility, initiates the data read program in that facility, and begins to send data.

In the data input mode, the keys and synchronizer channel are sampled and a clock is incremented every tenth second. Whenever a change in state in one or more of the keys or synchronizer occurs, the elapsed time from the nearest change and the switches that changed state are recorded. The procedure is continued until recording is terminated by releasing the time switch. Recorded data are transferred to cassette tape whenever the data buffer is full and at the end of a recording run. In the data transmission mode, the data is reread from the cassette tape into the data buffer. It is then transferred to the storage and analysis computer via a timesharing data port at approximately $300 \mathrm{bits} / \mathrm{sec}$.

The ELOG system weighs less than $10 \mathrm{lbs}$ including batteries. Cost of parts, including keyboards, is approximately $\$ 100$. Cost of the complete system, including

Table 1

Comparison of Features Found in Various Input Hardware

\begin{tabular}{|c|c|c|c|c|c|c|c|c|c|}
\hline \multirow[b]{2}{*}{ Feature } & \multirow{2}{*}{$\begin{array}{l}\text { Standard Com- } \\
\text { puter Data } \\
\text { Terminals } \\
\end{array}$} & \multicolumn{8}{|c|}{ Data Logging Input Devices } \\
\hline & & $\begin{array}{l}\text { Azur- } \\
\text { data }\end{array}$ & BOSS & ELOG & $\begin{array}{l}\text { Epic } \\
\text { Data }\end{array}$ & $\begin{array}{l}\text { Esterline } \\
\text { Angus }\end{array}$ & $\begin{array}{l}\text { Data- } \\
\text { myte }\end{array}$ & SSR6 & SSR7 \\
\hline Timekeeping & & & $\mathrm{x}$ & $\mathbf{X}$ & $\mathrm{x}$ & $\mathbf{x}$ & $\mathbf{x}$ & $\mathrm{x}$ & $\mathrm{x}$ \\
\hline $\begin{array}{l}\text { Entry Mode } \\
\text { Serial } \\
\text { Parallel }\end{array}$ & $\mathbf{X}$ & $\mathbf{x}$ & $\mathbf{x}$ & $\mathbf{x}$ & $\mathbf{X}$ & $\begin{array}{l}\mathbf{X} \\
\mathbf{X}\end{array}$ & $\mathbf{x}$ & $\begin{array}{l}\mathrm{X} \\
\mathrm{X}\end{array}$ & $\begin{array}{l}\mathrm{X} \\
\mathrm{X}\end{array}$ \\
\hline Paper Copy & Most & & & & & $\mathbf{X}$ & & & \\
\hline $\begin{array}{l}\text { Partability } \\
\text { Timesharing Accessible } \\
\text { Lightweight } \\
\text { Battery Operation }\end{array}$ & 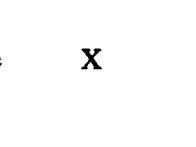 & $\begin{array}{l}\mathbf{X} \\
\mathbf{X} \\
\mathbf{X}\end{array}$ & $\begin{array}{l}\mathbf{X} \\
\mathbf{X}\end{array}$ & $\begin{array}{l}\mathbf{X} \\
\mathbf{X} \\
\mathbf{X}\end{array}$ & $\begin{array}{l}\mathbf{X} \\
\mathbf{X}\end{array}$ & $\mathbf{X}$ & $\begin{array}{l}\mathbf{X} \\
\mathbf{X} \\
\mathbf{X}\end{array}$ & $\begin{array}{l}X \\
X\end{array}$ & $\begin{array}{l}\mathbf{X} \\
\mathbf{X} \\
\mathbf{X}\end{array}$ \\
\hline Synchronization & & & & $\mathbf{x}$ & * & * & & & $x$ \\
\hline $\begin{array}{l}\text { Data Transmission Rate } \\
\text { High }(>300 \mathrm{bps}) \\
\text { Medium }(\approx 300 \mathrm{bps}) \\
\text { Low }(<300 \mathrm{bps})\end{array}$ & $\begin{array}{l}\mathbf{X} \\
\mathbf{X} \\
\mathbf{X}\end{array}$ & $\begin{array}{l}\mathbf{X} \\
\mathbf{X}\end{array}$ & $\mathbf{X}$ & $\mathbf{X}$ & $\begin{array}{l}\mathbf{X} \\
\mathbf{X}\end{array}$ & $\begin{array}{l}\mathbf{X} \\
\mathbf{X}\end{array}$ & $\begin{array}{l}\mathbf{X} \\
\mathbf{X}\end{array}$ & $\mathrm{X}$ & $\mathrm{X}$ \\
\hline $\begin{array}{l}\text { Data Storage } \\
\text { Electronic Memory } \\
\text { Computer Tape }\end{array}$ & $* *$ & $\mathrm{X}$ & & & $\mathbf{X}$ & $\begin{array}{l}\mathbf{X} \\
\mathbf{X}\end{array}$ & $\mathbf{x}$ & & \\
\hline $\begin{array}{l}\text { Audio Cassette } \\
\text { Punched Paper }\end{array}$ & & & $* * *$ & $\mathbf{X}$ & & $\mathrm{x}$ & & $* * *$ & $* * *$ \\
\hline
\end{tabular}

*Device has the capacity if an interface is built.

* Most Teletypes have paper tape punches and some data terminals contain tape or electronic memory.

***Reel-to-reel or cassette audio tapes run continuously and provide event timing. Limited time capacity. 
hardware and labor, ranges from $\$ 500$ to $\$ 1,000$. Detailed information may be obtained from the author.

\section{INPUT DEVICES}

Table 1 provides a comparison of some currently available data recording input devices. Addresses of the commercial input device companies cited in Table 1 are listed below.

\author{
Azurdata \\ P.O. Box 926 \\ Richland, Washington 99352 \\ Epic Data Corporation \\ 1272815 th Place, N.E. \\ Bellevue, Washington 90885 \\ Esterline Angus Instrument Corporation \\ Box 24000 \\ Indianapolis, Indiana 46224

\section{Electro/General Corporation (Datamyte) 14960 Minnetonka Industrial Road \\ Minnetonka, Minnesota 55343}

Detailed descriptions of BOSS and SSR6 may be found in Hollenbeck et al. (Note1) and Stephenson et al. (1975), respectively. The SSR7 system is described elsewhere in this issue of the Journal.

\section{REFERENCE NOTES}

1. Hollenbeck, A. R., Smythe, L. E., \& Sackett, G. P. BOSS. A manual for computer-assisted observational research at the University of Washington. Seattle: University of Washington, 1975.

2. Holm. R. A. Techniques of recording observational data. Report prepared for a conference on application of observational/ethological methods to the study of mental retardation, Lake Wilderness, Washington, 1976.

3. Bakeman, R. Untangling streams of behavior: Sequential analysis of obsenational data. Report prepared for a conference on application of observational/ethological methods to the study of mental retardation. Lake Wilderness, Washington, 1976.

\section{REFERENCES}

Fitzpatrick. L. J. BEHAVE: An automated data analysis system for observed events. Behavior Research Methods \& Instrumentation, 1977, 9, 452-455.

Hopf, S. Study of spontaneous behavior in squirrel monkey groups: Observation techniques, recording devices, numerical evaluation and reliability tests. Folia Primatologica. 1972. 17. $363-388$

Odom, B.. Mitchell, G., \& Lindburg, D. A device to record primate social behavior. Primates, 1970, 11. 93-96.

Sackett, G. P., Stephenson, E., \& Ruppenthal, G. D. Digital data acquisition systems for observing behavior in laboratory and field settings. Behavior Research Methods \& Instrumentation, 1973, 5, 344-348.

Stephenson, G. R., Smith, D. P. B., \& Roberts, T. W. The SSR system: An open format event recording system with computerized transcription. Behavior Research Methods \& Insirumentation, 1975, 7, 497-515.

WhITE. R. E. C. WRATS: A computer compatible system for automatically recording and transcribing behavioural data. Behaviour, 1971, 40,135-161.

(Received for publication August 16. 1977; revision accepted August 30, 1977.) 